\title{
A Local Knowledge Base for Service Oriented Access Network Selection
}

\author{
Carolina Fortuna \\ Jozef Stefan Institute \\ Jamova 39, 1000 Ljubljana \\ Slovenia \\ +38614773114 \\ carolina.fortuna@ijs.si
}

\author{
Mihael Mohorcic \\ Jozef Stefan Institute \\ Jamova 39, 1000 Ljubljana \\ Slovenia \\ +38614773669 \\ miha.mohorcic@ijs.si
}

\begin{abstract}
Service oriented access in a multi-application, multi-access network environment poses interesting research challenges. One of these challenges refers to cross-layer interoperability among technologies. In this poster, we introduce a knowledge base (KB) which contains local (user terminal specific) knowledge that enables pro-active network selection by translating technology specific parameters to higher-level, more abstract parameters. We implemented a prototype which makes use of semantic technology (namely ResearchCyc) for creating the elements of the KB: the ontology, the concepts, facts and rules. The system implements technologyspecific QoS parameters mapping according to the IEEE 802.21 draft standard recommendation.
\end{abstract}

\section{Categories and Subject Descriptors}

C.2.6 [Computer Communication Networks]: Network

Architecture and Design - Asynchronous Transfer Mode (ATM),

Centralized network, Circuit-switching networks, Distributed networks, Frame relay networks, ISDN (Integrated Services Digital Network), Network communications, Network topology, Packetswitching networks, Store and forward networks, Wireless communication.

General Terms; Design, Management, Standardization.

\section{Keywords}

Vertical handover, knowledge base, service oriented, network selection.

\section{INTRODUCTION}

Mobile terminals such as notebooks, internet tablets, smart phones and, recently, netbooks [1] are dramatically increasing in numbers and already tend to replace desktop computers as the primary connectivity device. The problem with all these portable devices is that they have limited computing capacity. As a consequence, they are mainly used for web browsing, email, multimedia consumption and generation, or simply connecting to a virtual machine running somewhere in the computing cloud and performing more intensive computation. All these usage patterns require connectivity, especially wireless connectivity.

Copyright is held by the author/owner(s)..

Mobiheld'09, August 17, 2009, Barcelona, Spain.

ACM 978-1-60558-444-7/09/08...\$10.00.
Although mobile terminals today feature interfaces for at least two wireless technologies (i.e. UMTS, WiFi), connecting via the "best" access technology is still not possible. This freedom of choice is bounded by technological as well as business related constraints. Two major standards organizations, IEEE and 3GPP, are working on drafts (IEEE 802.21 and 3GPP UMA, GAN, VCC) to standardize handover between heterogeneous access networks, also called vertical handover [2].

By service oriented access network selection we refer to selecting a target network for (1) connecting to or (2) handing over to in a manner that allows consuming electronic services with high quality of experience. When applications running on the mobile terminal detect that the user might experience degradation in the quality of experience caused by the access segment, a network selection for handover process starts. The optimum would be a transparent layer3 handover which preserves the state (including IP address, port numbers and security associations) of the deteriorating connections [2].

A handover can be requested by the mobile terminal (mobile initiated handover) or by the network (network initiated handover). Vertical handovers have not yet become a standard feature, but there are several efforts under way in this direction as summarized in the following.

In this paper, we introduce a local knowledge base (KB) for proactive network selection choosing between IEEE 802.11, 802.16 and UMTS. As opposed to other similar works in the literature, our approach makes use of a KB (ontology + facts + rules + instances), where access interfaces publish specific technology dependent information, which can be queried by high layer entities. The KB handles all the parameter translations from low level technology dependent to high level, technology and platform independent ones. To the best of our knowledge, this is the first attempt to use a KB approach for network selection purposes in wireless communications.

\section{SEMANTIC MAPPING OF QOS PARAMETERS}

Provisioning users with desired application experience requires the network selection system to take into account QoS related parameters and their mapping between services at different layers of the protocol stack, particularly between IP QoS and link layer QoS. ITU has specified five QoS parameters for IP transport of applications: transfer delay, delay variation, loss ratio, error rate and throughput. Based on these parameters, 6 classes of service for 
packet classification have been defined; these are numbered from 0 to $1[4]$.

Early IEEE 802.11 (WiFi) specifications do not define any mechanisms for QoS support. However, starting with the IEEE 802.11e revision, QoS support with 4 classes of service (for EDCA) [3] has been added. This version of the standard is also referred to as wireless multimedia (WMM). Later, the $802.11 \mathrm{k}$ amendment also introduced mechanisms for measuring relevant radio link layer parameters. Handover capability among access points interconnected at the link layer is provided by two amendments to the standard, 802.11f, which allows access points to exchange information and $802.11 \mathrm{r}$, which deals with "fast" handover, i.e. handover taking QoS into account [4].

IEEE 802.16 (WiMAX) defined four classes of service and the 802.16e amendment to this standard introduced the fifth, called ertPS. Finally, 3GPP defines 4 QoS classes, conversational, streaming, interactive and background.

There are three types of QoS-related information which can be shared among different technologies using the Media Independent Handover Function (MIHF) [4]:

- Service classes

- QoS parameters (per service class)

- Network performance measurements (per service class).

In our implementation, we introduced in the $\mathrm{KB}$ concepts related to service classes, QoS parameters, relations between these, and rules for cross-standard mapping between IEEE 802.11, 802.16, 802.21 and UMTS. These mappings can be found in tables in [4] and Annex I of [3]. Figure 1 shows how MIH users can use the MIHF to query the local $\mathrm{KB}$ in which interface specific knowledge and parameters values are published.

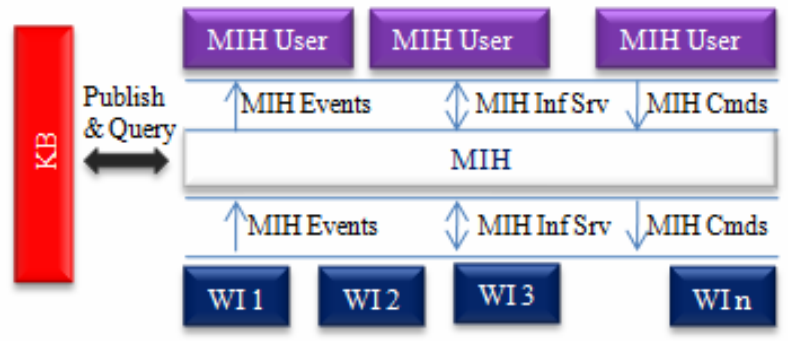

Figure 1 Local KB for service oriented network selection

\section{MODELLING THE LOCAL KNOWLEDGE BASE}

Two of the basic rules of ontology engineering refer to inserting concepts and to inserting predicates, functions and rules [5]. A concept in an ontology should be as specific as possible, so it should be inserted as low as possible in the taxonomy. Predicates, functions and rules, on the other hand, should be as general as possible, thus referring to as many concepts as possible while being consistent. In our approach, we tried to follow these rules as close as possible.
However, since we are modeling domain specific knowledge in a microtheory, our rules are quite specific.

Two of the most relevant concepts we introduced in the $\mathrm{KB}$ are AccessNetwork (as a specialization of the ComputerNetwork concept) and ClassOfService, while some instances of protocols are IEEE802dot11Protocol, IEEE802dot16Protocol, 3GPPProtocol and IEEE802dot21Protocol. Having concepts inserted in the ontology, we needed a mechanism to assert that an instance of AccessNetwork, e.g. WiFil, implements the IEEE802dot11Protocol and to specify the version of the implemented protocol (i.e. "a", "g", "b", etc.). Predicates such as networkImplementsProtocol and implementsVersionOfProtocol can be used for this.

After inserting taxonomical information, the next step was to create rules for mapping IEEE 802.21 specific QoS parameters and classes of service to those corresponding to the underlying technologies according to the implemented protocol.

Finally, the result of extending the $\mathrm{KB}$ and creating instances, enables a MIH user to issue queries and receive correct answers. An example query expressed in $\mathrm{CycL}$ is presented in Figure 2 and can be translated to natural language as: "Which are the published networks which have maximum packet transfer delay more than 0.01 seconds?".

(\#\$and

(\#\$hasCoSMaxPacketTransferDelay-802dot21 ?X ?Y)

(\#\$greaterThan ?Y

(\#\$SecondsDuration 0.1)))

Figure 2 Example of a rule in the local $\mathrm{KB}$

\section{CONCLUSIONS}

This paper introduced a local KB implemented using ResearchCyc. The KB enables pro-active network selection for service oriented wireless access networks. We briefly described the implementation of the $\mathrm{KB}$ and discussed a form of knowledge representations suitable for this.

\section{REFERENCES}

[1] C. Thompson, "The Netbook Effect: How Cheap Little Laptops Hit the Big Time", Wired Magazine, 17 March 2009.

[2] J. Fabini, R. Pailer and P. Reichl, "Location-based assisted handover for the IP Multimedia Subsystem", Computer Communications, Vol. 31, Issue 10, 25 June 2008, pp. 23672380.

[3] "IEEE P802.21/D11.0 Draft Standard for Local and Metropolitan Area Networks: Media Independent Handover Services", May 2008.

[4] D. J. Wright, "Maintaining QoS During Handover Among Multiple Wireless Access Technologies", Int. Conf. on the Management of Mobile Business, 2007.

[5] C. Matuszek, J. Cabral, M. Witbrock, and J. DeOliveira, "An introduction to the syntax and content of cyc", AAAI 2006, AAAI Press, Menlo Park, CA, 4449, 2006. 